\title{
Protracted febrile myalgia syndrome in patient with familial mediterranean fever
}

\begin{abstract}
Familial Mediterranean fever (FMF) is a one of genetically basis auto inflammatory disease characterized by recurrent episodes of fever, serositis \& musculoskeletal involvement. Protracted febrile myalgia syndrome (PFMS) is characterized by severe paralyzing myalgia, high fever, abdominal pain, diarrhea, arthritis/arthralgia, and transient vasculitic rashes. We report a 15 year-old girl with PFM as non classical presenting symptoms of FMF, and discuss the course of management of this case.

We reviewed the literature and mostly recorded all case reports of PFM affected adolesecent and adult patients over the past years to give special prominence to muscle affection in FMF particularly PFM
\end{abstract}

Keywords: FMF, protracted febrile myalgia, auto inflammatory, fever
Volume 8 Issue 2 - 2018

\author{
Manal El Menyawi,' Mervat Essam, ${ }^{2}$ Ahmed \\ Shaker, ${ }^{3}$ Fadi Nagi ${ }^{3}$ \\ 'Professor of Internal medicine, Cairo University, Egypt \\ ${ }^{2}$ Lecturer of Internal medicine, Cairo University, Egypt \\ ${ }^{3}$ Resident of Internal medicine, Cairo University, Egypt
}

Correspondence: Mervat Essam, Cairo University, Egypt, Tel +20 01 I 2024507I, Email lambaa2020@yahoo.com

Received: February 10, 2018 | Published: March 19, 2018

\section{Introduction}

Familial Mediterranean fever is a disease described by paroxysmal and self limiting episodes of fever, serositis \& synovitis. Sephardic Jews, Armenians, Turkish, and Arabs are predominantly affected through a recessive autosomal mode of transmission. ${ }^{1}$

Myalgia is not uncommon musculoskeletal manifestations (occurs in 20\%). Protracted febrile myalgia (PFMS), one of muscular pain disorder characterized by severe disabling myalgia, high fever, abdominal pain, diarrhea, arthritis/arthralgia. ${ }^{2}$

High erythrocyte sedimentation rate (ESR), hyperglobulinemia, normal creatinine phosphokinase (CPK), and leukocytosis are the other findings. ${ }^{3}$ The episodes last for 4-6 weeks, except in those patients need to be treated with corticosteroids. The PFMS may recur even under colchicine prophylaxis. In this article we describe our case, and also collect \& summarize all reported cases from searching on the related references and reviews of literature

\section{Case Report}

15 yrs old female patient born and lives in Kafr Elsheikh, Egypt was diagnosed to have typical attacks of FMF 5 years ago on colchicine treatment $(1.2 \mathrm{mg} / \mathrm{d})$, Molecular genetic study revealed positive heterozygous state, MEFV gene mutations (M694V , E148Q , P369S). The patient has positive family history (her father and uncle have FMF), During the past 6 months, the patient experienced bilateral diffuse, non exertional crippling muscle ach with swelling of both thighs restricting movement of lower limbs, not relieved by colchicine or NSAIDS, the condition was not associated with fever and colicky abdominal pain or skin rash for which she was admitted in the hospital of the faculty of medicine Alex University where initial examination and investigations were done then the patient sought a medical advice in our department. On systematic physical review: The well-developed patient was bedridden in agony normal blood pressure and temperature. Height $=145 \mathrm{cms}$, weight $=40 \mathrm{kgs}$, $\mathrm{BMI}=19$. There was bilateral symmetrical diffuse \& tender swelling of both thighs with subcutaneous edema made her unable to move the lower limbs properly, sensory and motor examination of the upper extremities was normal, Cardiac, chest, abdominal examination was apparently free. Laboratory investigations revealed normal levels of CK, CK-MB, Aldolase \& transaminases. Hemoglobin $13.8 \mathrm{~g} / \mathrm{dL}$; white blood cell count 5000/mm3, platelet count, 209,000/mm3; ESR was $14 \mathrm{~mm} / \mathrm{h}$ and high $\mathrm{C}$-reactive protein $(\mathrm{CRP})(40 \mathrm{Iu} / \mathrm{L})$. Normal liver \& kidney function tests. ASOT within normal range $(150 \mathrm{U} / \mathrm{ml})$, ANA, ANCA, RF, serum amyloid A\& virology markers for HCV, HBV\& CMV were negative Chest X-ray, Abdominal ultrasonography, bilateral lower limb arterial and venous duplex \& Ultrasound knee joint showed no abnormalities. MRI of both thigh on presentation showed right posterolateral thigh ill defined patchy area of soft tissue edema involving subcutaneous tissue eliciting high signal in STIR \& measured about $10 \mathrm{~cm}$, normal MR musculature appearance with intact intermuscular fascial fat planes (Figure 1)(Figure 2).

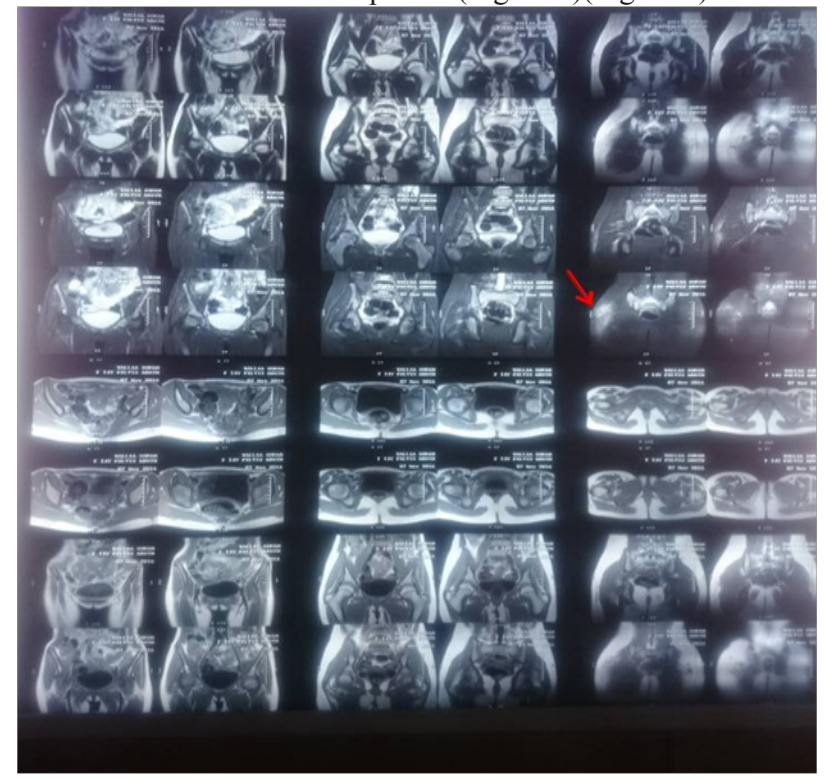

Figure I Intermuscular fascial fat planes. 


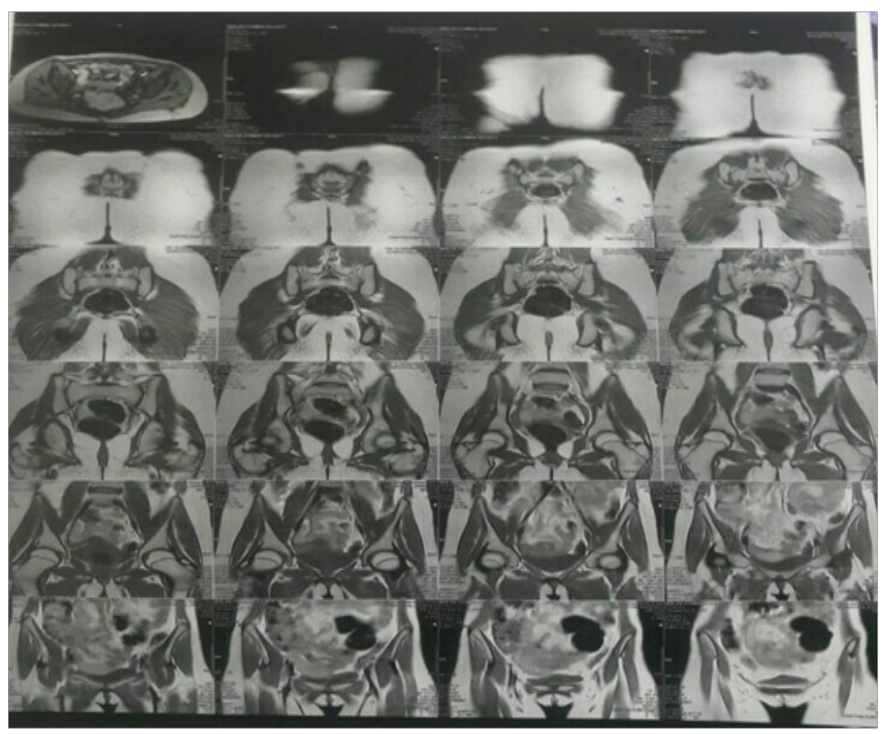

Figure 2 Electromyogram (EMG) and sensorimotor nerve conduction studies of both lower limbs showed evidence of patchy myopathy.

Electromyogram (EMG) and sensorimotor nerve conduction studies of both lower limbs showed evidence of patchy myopathy. After exclusion the presence of vasculitis which mostly associated with FMF and myalgia induced colchicine; we assumed that the patient had porotracted febrile myalgia (PFM), so pridnosolone $0.5 \mathrm{mg} / \mathrm{Kg}$ was first prescribed but the patient did not improve then we escalate the dose to $1 \mathrm{mg} / \mathrm{kg} / \mathrm{d}$ with extensive physiotherapy for more than 2 months the clinical condition was ameliorated (the Subcutaneous edema \& muscle pain subsided \& the patient could independently walk) and follow up labs were improved regarding CRP (4 IU/L). Follow up MRI showed no abnormality

Then we started to tapper steroids slowly

\section{Discussion}

Familial Mediterranean fever (FMF) is a genetic disease characterized by painful febrile attacks of serositis and systemic manifestations. Since the disease first described in 1945 and the gene of the disease identified in 1992, many sets of criteria were described for the diagnosis. The commonest of these is Tel Hashomer's (Table 1) depending on major and minor criteria. ${ }^{4}$ The pathogenesis of FMF $^{5}$ is related to exaggerated inflammatory response secondary to abnormal pyrin that has a critical role in the regulation of IL-1 pathway and mutations result in increased IL-1 production, which is believed to be responsible for the clinical characteristics. During episodes, the chemotactic activity of the granulocytes is greatly increased with a massive influx of granulocytes to the affected tissues. ${ }^{6}$ Pyrin is expressed only in granulocytes and is likely to be a nuclear factor that controls the inflammatory response in differentiated granulocytes.

The gene responsible for FMF, MEFV, was identified in $1992^{7}$ and nearly 30 mutations have subsequently been detected. ${ }^{8}$ The most common are M694V and V726A. Variable MEFV mutations have different diagnostic, therapeutic, and prognostic implications. ${ }^{9}$

Streptococci might be assumed to be the incriminating agents due to increased ASO titers, circulating immune complexes, hypergammaglobulinemia, and complement component consumption are detected, in patients ${ }^{10}$ especially those with vasculitis. ${ }^{11}$
Table I Criteria for FMF diagnosis

\begin{tabular}{|c|c|}
\hline Major criteria & Minor criteria \\
\hline I. Peritonitis, & Incomplete attacks of chest, \\
\hline 2. Pleuritis or pericarditis, & $\begin{array}{l}\text { Incomplete attacks of joint } \\
\text { (joints other than specified } \\
\text { in major criteria) }\end{array}$ \\
\hline
\end{tabular}

3. Monoarthritis (hip, knee, ankle), Exertional leg pain and

4. Fever alone and

5. Incomplete abdominal attacks

Favorable response to colchicine.

As presented the most important musculoskeletal symptoms is joint involvement, however muscle pain and tenderness may be featured, It has been reported as $20-25 \%$ in patients with FMF and has various forms of including myalgia related to Henoch Schönlein purpura and Polyarteritis nodosa, exertional leg pain, fibromyalgia, constitutional generalized myalgia \& finally colchicine induced myopathy. ${ }^{2}$

Protracted febrile myalgia (PFM) is a rare pattern (1.2\%) of myalgia in FMF. However it may be the first presentation of FMF. ${ }^{12}$ It is characterized by episodes of prolonged incapacitating muscle pain $\&$ tenderness lasting up to 6 weeks. ${ }^{3}$ It is associated by high fever, abdominal pain, diarrhea, arthritis/arthralgia, vasculitic rash, and nephritis. Most of the patients with PFMS bear M694V mutation, However there is a report of PFM case homozygous for E148Q. ${ }^{13}$ These attacks are not prevented by colchicine and do not respond to non-steroidal anti-inflammatory drugs. Muscle enzymes are within normal limits with elevated erythrocyte sedimentation rate, hyperglobulinemia \& leukocytosis. ${ }^{14}$

MRI can be considerd as a valuable tool to diagnose PFMS, the features that suggest PFMS are diffuse edema of subcutaneous fat tissue, increased intensity and contrast enhancement of the muscles and increased signal intensity of vascular beds. ${ }^{15}$ Electromyogram (EMG) may be normal or show nonspecific myopathic changes, Muscle biopsy was not feasible in most of diagnosed cases, but of which was done revealed non specific inflammatory infiltration with leucocytes, lymphocytes and eosinophills.

It was peculiar that our young lady case presented with atypical attack of FMF consisting of severe muscle pain \& swelling affecting both thigh but not accompanied by fever, rash or abdominal pain. Also ESR was not elevated as excepected there is no definite rational for that; yet the compliance of the patient to colchicine may abort the elevation of ESR as acute phase reactant. We began to revise the history and to put differential diagnosis. Myopathy related to FMF was on the top after exclusion of vasculitis. Hence Fulfiling the clinical criteria, normal CPK levels \& characteristic MRI findings (oedema of subcutaneous fat tissue) together with the evidence of inflammatory myopathy by EMG are the hallmarks of diagnosing PFMS and ruling out the colchicine induced myopathy. Immediately, the decision was made based on the foregoing \& Steriods were prescribed with collaporation of physiotherapy team for more than 2 months. A good response was achieved and the patient was able to move independently

PFM was first described by Schwabe et al. ${ }^{16}$ They noticed the attacks of fever and severe myalgia lasting 10-21 days. In 1988 Schapira et al. ${ }^{16}$ described a patient whose only manifestation of FMF was severe myalgia with fever. ${ }^{17}$ In 1994 Langevitz et al ${ }^{17}$ described 14 FMF patients with severe disabling myalgia accompanied by fever, high erythrocyte sedimentation rate, lasting up to 6 weeks. Transient 
vasculitic rashes were reported in few cases.

the M694V mutation and PFM Kaplan et al defined a group of criteria

Kaplan and co decided that there is a strong association between for the diagnosis of PFM in a group of children in 2007..$^{18}$

Table 2 Adult PFA

\begin{tabular}{|c|c|c|c|c|c|c|c|c|c|c|c|}
\hline Number & $\begin{array}{l}\text { Agel } \\
\text { Sex }\end{array}$ & FMF & $\begin{array}{l}\text { Sym } \\
\text { ptoms }\end{array}$ & Labs & MRI & EMG & Biopsy & FMF gene & $\begin{array}{l}\text { Typical } \\
\text { FMF }\end{array}$ & $\begin{array}{l}\text { Famiy } \\
\text { H/O }\end{array}$ & $\begin{array}{l}\text { Treatment } \\
\text { and } \\
\text { outcome }\end{array}$ \\
\hline $\begin{array}{l}\text { I Bircan } \\
\text { Z \& } \\
\text { Usluer } \\
\mathrm{H}^{28}\end{array}$ & $\begin{array}{l}15 \\
1 \mathrm{M}\end{array}$ & Known & $\begin{array}{l}\text { Abdpain, } \\
\text { vomiting, } \\
\text { fever and } \\
\text { dehydration } \\
\text { Myalgia in } \\
\text { prox. Ms. I3 } \\
\text { yrs ago }\end{array}$ & $\begin{array}{l}\text { Slight Inc ALT, } \\
\text { AST } \\
\text { Normal CK } \\
\text { CRP }=12.7 \\
\text { ESR }=115 \\
\text { ASOT }=303 \\
\text { Virology -ve } \\
\text { ANA,P- } \\
\text { ANCA-ve }\end{array}$ & & & & $\begin{array}{l}\text { +ve homogenus } \\
\text { for } \\
\text { M694V } \\
\text { mutation }\end{array}$ & typical & $+v e$ & $\begin{array}{l}\text { To } \\
\text { colchicine } \\
\text { and } \\
\text { prednisolone } \\
2 \mathrm{mg} / \mathrm{kg} \\
\text { Follow up : } \\
\text { normalization } \\
\text { of acute } \\
\text { phase } \\
\text { reactant }\end{array}$ \\
\hline $\begin{array}{l}\text { 2Bircan Z } \\
\text { \& Usluer } \\
\mathrm{H}^{28}\end{array}$ & $12 / F$ & Known & $\begin{array}{l}\text { fever, } \\
\text { abdominal } \\
\text { pain and } \\
\text { myalgia }\end{array}$ & $\begin{array}{l}\text { Normal ALT, } \\
\text { AST,ALP,CK } \\
\text { Normal } \\
\text { C3,C4 } \\
\text { ESR= } \\
\text { II } 3 \mathrm{~mm} / \mathrm{h} \text {, } \\
\text { CRP26.6 } \\
\mathrm{mg} / \mathrm{DI} \\
\text { Leucocytosis } \\
\text { Serology } \\
\text { for hepatitis } \\
\text { and lupus -ve } \\
\text { ANCA -ve }\end{array}$ & & normal & & & typical & +ve & $\begin{array}{l}\text { prednisolone } \\
2 \mathrm{mg} / \mathrm{kg} \\
\text { Follow up : } \\
\text { normalization } \\
\text { of acute } \\
\text { phase } \\
\text { reactant }\end{array}$ \\
\hline $\begin{array}{l}3 \text { Schapira } \\
D \text { et al. }{ }^{16}\end{array}$ & $22 / \mathrm{F}$ & $\begin{array}{l}\text { Not } \\
\text { known }\end{array}$ & $\begin{array}{l}\text { Arthritis } \\
\text { of ankle, } \\
\text { knee joints } \\
\text { I6 yrs ago } \\
\text { Sever pain } \\
\text { in calf mscs } \\
\text { with fever }\end{array}$ & $\begin{array}{l}\text { Normal ALT, } \\
\text { AST,ALP, CK, } \\
\text { aldolase -ve } \\
\text { ANA, ASMA, } \\
\text { AMA and } \\
\text { RFCRP+++ } \\
\text { ESR=80 }\end{array}$ & & normal & $\begin{array}{l}\text { histological:inc } \\
\text { in collagen } \\
\text { tissue in } \\
\text { interstitium } \\
\text { with most msc } \\
\text { fiber of normal } \\
\text { size E/M: most } \\
\text { muscle fibres } \\
\text { were of normal } \\
\text { size with some } \\
\text { atrophic cells }\end{array}$ & & Atypical & +ve & $\begin{array}{l}\text { No } \\
\text { response to } \\
\text { prednisolone } \\
\text { alone } \\
\text { Improved on } \\
\text { colchicine } \\
1.5 \mathrm{mg} / \mathrm{kg} \\
\text { Follow up : } \\
\text { normalization } \\
\text { of acute } \\
\text { phase } \\
\text { reactant }\end{array}$ \\
\hline $\begin{array}{l}3 \\
\text { Kotevoglu } \\
\mathrm{N} \text { et al. }{ }^{29}\end{array}$ & $13 \mathrm{~F}$ & $\begin{array}{l}\text { Not } \\
\text { known }\end{array}$ & $\begin{array}{l}\text { Sever RT } \\
\text { leg and } \\
\text { knee ms } \\
\text { pain with } \\
\text { fever } 3 \\
\text { months } \\
\text { inability to } \\
\text { walk }\end{array}$ & $\begin{array}{l}\text { Normal ALT, } \\
\text { AST, ALP } \\
\text { Leucocytosis } \\
\text { Elevated ESR, } \\
\text { CRP and CK }\end{array}$ & $\begin{array}{l}\text { oedema } \\
\text { of the } \\
\text { subcutaneous } \\
\text { fat tissue and } \\
\text { the distal part } \\
\text { of the medial } \\
\text { gastrocnemius } \\
\text { muscle } \\
\text { prior to the } \\
\text { musculo- } \\
\text { cutaneous } \\
\text { junction of } \\
\text { the Achilles } \\
\text { tendon }\end{array}$ & normal & $\begin{array}{l}\text { Scarce non } \\
\text { specific } \\
\text { inflamma-tory } \\
\text { inflilterat-ion } \\
\text { with leucocyte, } \\
\text { lymphocytes } \\
\text { and eosinophils }\end{array}$ & $\begin{array}{l}+ \text { ve } \\
\text { hetregonous for } \\
\text { M694V } \\
\text { andV726A }\end{array}$ & Atypical & & $\begin{array}{l}\text { Respond to } \\
\text { steroids and } \\
\text { colchicines } \\
\text { Follow up : } \\
\text { normalization } \\
\text { of acute } \\
\text { phase } \\
\text { reactant }\end{array}$ \\
\hline $\begin{array}{l}4 \\
\text { ALPAYCI } \\
M \text { et al. }{ }^{21}\end{array}$ & $33 M$ & $\begin{array}{l}\text { Not } \\
\text { known }\end{array}$ & $\begin{array}{l}\text { Sever } \\
\text { paralyizing } \\
\text { myalgia, abd } \\
\text { pain, fever, } \\
\text { muscle } \\
\text { tendrness }\end{array}$ & $\begin{array}{l}\text { Normal ALT, } \\
\text { AST, CK, } \\
\text { ASOT, RF } \\
\text {-ve virology } \\
\text { Leukocytosis } \\
\text { ESR=36 } \\
\text { CRP=5I }\end{array}$ & & & & $\begin{array}{l}\text { +ve } \\
\text { heterozygous } \\
\text { V726A }\end{array}$ & typical & & $\begin{array}{l}\text { Respond to } \\
\text { steroids and } \\
\text { colchicines } \\
\text { Follow up : } \\
\text { normalization } \\
\text { of acute } \\
\text { phase } \\
\text { reactant }\end{array}$ \\
\hline
\end{tabular}


Table Continued.....

\begin{tabular}{|c|c|c|c|c|c|c|c|c|c|c|c|}
\hline Number & $\begin{array}{l}\text { Agel } \\
\text { Sex }\end{array}$ & FMF & $\begin{array}{l}\text { Sym } \\
\text { ptoms }\end{array}$ & Labs & MRI & EMG & Biopsy & FMF gene & $\begin{array}{l}\text { Typical } \\
\text { FMF }\end{array}$ & $\begin{array}{l}\text { Famiy } \\
\text { H/O }\end{array}$ & $\begin{array}{l}\text { Treatment } \\
\text { and } \\
\text { outcome }\end{array}$ \\
\hline $\begin{array}{l}5 \text { Tufan G } \\
\text { \& Demir } \\
S^{23}\end{array}$ & $30 \mathrm{~F}$ & known & $\begin{array}{l}\text { Sever } \\
\text { diffuse ms } \\
\text { pain and } \\
\text { tenderness } \\
\text { For } 2 \\
\text { months }\end{array}$ & $\begin{array}{l}\text { Normal CK, } \\
\text { ALT, AST } \\
\text { leucocytosis } \\
\text {-ve ANA, } \\
\text { antiDNA, } \\
\text { ANCA } \\
\text { ESR }=140 \\
\text { CRP }=158\end{array}$ & & & & & & & $\begin{array}{l}\text { No response } \\
\text { to colchicine } \\
\text { alone } \\
\text { Respond } \\
\text { to added } \\
\text { steroids, } \\
\text { tappered } \\
\text { then stopped } \\
\text { Follow up : } \\
\text { normalization } \\
\text { of acute } \\
\text { phase } \\
\text { reactant }\end{array}$ \\
\hline $\begin{array}{l}6 \\
\text { Fujikawa } \\
\text { Ket al. }{ }^{130}\end{array}$ & $22 M$ & known & $\begin{array}{l}\text { Fever, } \\
\text { prolonged } \\
\text { myalgia of } \\
\text { both UL } \\
\text { and LL, abd } \\
\text { pain, } \\
\text { diarrhea } \\
\text { For } 3 \\
\text { weeks }\end{array}$ & $\begin{array}{l}\text { Normal CK } \\
\text { Aldolase=6.6 } \\
\text { high ESR } \\
\& \text { CRP } \\
\text { ferritin=566 } \\
\text {-ve virology }\end{array}$ & $\begin{array}{l}\text { Thickening } \\
\text { of fascia } \\
\text { in STIR } \\
\text { suggestive } \\
\text { of Fasciitis }\end{array}$ & & $\begin{array}{l}\text { striking changes } \\
\text { appear in the } \\
\text { fascia, including } \\
\text { an increased } \\
\text { amount of } \\
\text { collagen fibrils } \\
\text { and infiltration } \\
\text { by fibroblasts, } \\
\text { macrophages } \\
\text { and a few mast } \\
\text { cells }\end{array}$ & $\begin{array}{l}\text { +ve } \\
\text { homozygous } \\
\text { for EI48Q and } \\
\text { compound } \\
\text { heterogenous } \\
\text { P3695-R408Q }\end{array}$ & typical & 更 & $\begin{array}{l}\text { Respond } \\
\text { rapidly to } \\
\text { small dose } \\
\text { steroid } \\
20 \mathrm{mg} / \mathrm{d} \text { and } \\
\text { discontinued } \\
\text { along } 6 \\
\text { weeks } \\
\text { Follow up : } \\
\text { normalization } \\
\text { of acute } \\
\text { phase } \\
\text { reactant }\end{array}$ \\
\hline $\begin{array}{l}7 \text { Ertekin } \\
\text { et al. }{ }^{31}\end{array}$ & $14 M$ & known & $\begin{array}{l}\text { Fever,abd } \\
\text { pain, sever } \\
\text { myalgia all } \\
\text { mscs For } \\
25 \text { days }\end{array}$ & $\begin{array}{l}\text { Normal } \\
\text { ALT,AST,CK } \\
\text {-ve ANA } \\
\text { ESR }=92 \mathrm{~mm} / \mathrm{h} \\
C R P=15 \mathrm{mg} / \mathrm{dl}\end{array}$ & & & & $\begin{array}{l}\text { +ve } \\
\text { heterozygous } \\
\text { mutation } \\
\text { EI48Q and } \\
\text { R76I }\end{array}$ & typical & $+\mathrm{ve}$ & $\begin{array}{l}\text { Respond } \\
\text { to steroids } \\
\text { within } \\
10 \text { days } \\
\text { Follow up : } \\
\text { normalization } \\
\text { of acute } \\
\text { phase } \\
\text { reactant }\end{array}$ \\
\hline $\begin{array}{l}8 \text { Rıdvan } \\
\text { M et al. }{ }^{32}\end{array}$ & $4 \mathrm{IF}$ & $\begin{array}{l}\text { Known } \\
2 \text { yrs } \\
\text { ago }\end{array}$ & $\begin{array}{l}\text { severe calf } \\
\text { and thigh } \\
\text { pain, fever } \\
\text { and malaise } \\
4 \text { weeks } \\
\text { duration }\end{array}$ & $\begin{array}{l}\text { Normal } \\
\text { ALT,AST,CK } \\
\text { Leucocytosis } \\
\text { ESR }=84 \\
\text { CRP }=178\end{array}$ & $\begin{array}{l}\text { MRI } \\
\text { revealed } \\
\text { remarkable } \\
\text { muscle } \\
\text { oedema } \\
\text { in her calf } \\
\text { muscles }\end{array}$ & & & $\begin{array}{l}\text { heterozygous } \\
\text { M694V } \\
\text { mutation }\end{array}$ & typical & & $\begin{array}{l}\text { Steroid } \\
\text { +colchicine } \\
\text { recurrence } \\
\text { anaqkinra } \\
100 \mathrm{mg} / \text { day } \\
\text { s.c response } \\
\text { Follow up : } \\
\text { normalization } \\
\text { of acute } \\
\text { phase } \\
\text { reactant }\end{array}$ \\
\hline $\begin{array}{l}9 \text { Ridvan } \\
\text { M et al. }{ }^{132}\end{array}$ & $44 \mathrm{~F}$ & $\begin{array}{l}\text { Known } \\
27 y r s \\
\text { ago }\end{array}$ & $\begin{array}{l}\text { severe right } \\
\text { thigh pain } \\
\text { for } 3 \text { weeks. } \\
\text { She also } \\
\text { complained } \\
\text { of } \\
\text { diarrhoea } \\
\text { and ankle } \\
\text { pain }\end{array}$ & $\begin{array}{l}\text { Normal } \\
\text { ALT,AST,CK } \\
E S R=78 \\
C R P=66\end{array}$ & & & & $\begin{array}{l}\text { homozygous } \\
\text { for M694V } \\
\text { mutation }\end{array}$ & typical & & $\begin{array}{l}\text { Naproxen } \\
\text { +colchicine } \\
\text { no response } \\
\text { Anakinra } \\
100 \mathrm{mg} / \text { day } \\
\text { s.c response } \\
\text { Follow up: } \\
\text { normalization } \\
\text { of acute } \\
\text { phase } \\
\text { reactant }\end{array}$ \\
\hline $\begin{array}{l}\text { IOSenel K } \\
\text { et al. } .^{33}\end{array}$ & $12 / M$ & $\begin{array}{l}\text { Known } \\
\text { to be } \\
\text { FMF }\end{array}$ & $\begin{array}{l}\text { LL pain } \\
\text {, skin } \\
\text { rash,fever } \\
\text { inability to } \\
\text { walk }\end{array}$ & $\begin{array}{l}\text { Normal } \\
\text { muscle } \\
\text { enzymes } \\
\text { CRP }=12 \\
\text { ESR }=108 \\
\text { ASOT=60I } \\
\text { Leukocytosis } \\
\text { Virology -ve }\end{array}$ & & $\begin{array}{l}\text { Non- } \\
\text { spesific } \\
\text { changes }\end{array}$ & & $\begin{array}{l}\text { homozygous } \\
\text { for M694V } \\
\text { mutation }\end{array}$ & & & $\begin{array}{l}\text { Steriodes } \\
\text { for } 3 \text { weeks } \\
\text { Follow up: } \\
\text { normalization } \\
\text { of acute } \\
\text { phase } \\
\text { reactant }\end{array}$ \\
\hline
\end{tabular}


Table Continued.....

\begin{tabular}{|c|c|c|c|c|c|c|c|c|c|c|c|}
\hline Number & $\begin{array}{l}\text { Agel } \\
\text { Sex }\end{array}$ & FMF & $\begin{array}{l}\text { Sym } \\
\text { ptoms }\end{array}$ & Labs & MRI & EMG & Biopsy & FMF gene & $\begin{array}{l}\text { Typical } \\
\text { FMF }\end{array}$ & $\begin{array}{l}\text { Famiy } \\
\mathrm{H} / \mathrm{O}\end{array}$ & $\begin{array}{l}\text { Treatment } \\
\text { and } \\
\text { outcome }\end{array}$ \\
\hline $\begin{array}{l}\text { II Soylu } \\
\text { et al. }{ }^{13}\end{array}$ & $14 / F$ & $\begin{array}{l}\text { Not } \\
\text { known } \\
\text { tohave } \\
\text { FMF }\end{array}$ & $\begin{array}{l}\text { UL \& LL } \\
\text { myalgia, } \\
\text { fever \& } \\
\text { arthralgia }\end{array}$ & 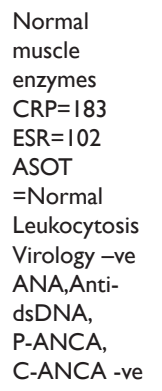 & & & & $\begin{array}{l}\text { homozygous } \\
\text { EI48Q }\end{array}$ & atypical & & $\begin{array}{l}\text { Improved } \\
\text { after } 24 \text { hrs } \\
\text { from intake } \\
\text { of steroids } \\
\text { prednisolone } \\
2 \mathrm{mg} / \mathrm{kg} / \mathrm{day} \\
\text { Follow up: } \\
\text { normalization } \\
\text { of acute } \\
\text { phase } \\
\text { reactant }\end{array}$ \\
\hline $\begin{array}{l}12 \text { Brican } \\
Z^{22}\end{array}$ & $12 / F$ & Known & $\begin{array}{l}\text { Abd pain } \\
\text { fever } \\
\text { Vomiting } \\
\text { diarrhea } \\
\text { Sever } \\
\text { myalgia } \\
\text { Skin rash } \\
\text { convulsion }\end{array}$ & $\begin{array}{l}\text { Normal } \\
\text { muscle } \\
\text { enzymes } \\
\text { leucocytosis } \\
\text { ESR=I15 } \\
\text { CRPI3.3 } \\
\text { ASOT: normal } \\
\text { ANA,ANCA } \\
\text {-veVirology } \\
\text {-ve }\end{array}$ & & Normal & & $\begin{array}{l}\text { homozygous for } \\
\text { the M694V }\end{array}$ & typical & & $\begin{array}{l}\text { prednisolone } \\
2 \mathrm{mg} / \mathrm{kg} \text { or } \\
\text { more than } \\
62 \text { days } \\
\text { Follow up: } \\
\text { normalization } \\
\text { of acute } \\
\text { phase } \\
\text { reactant }\end{array}$ \\
\hline $\begin{array}{l}\text { The } \\
\text { patient } \\
\text { of this } \\
\text { report }\end{array}$ & $15 / F$ & Known & $\begin{array}{l}\text { Severe } \\
\text { bilateral } \\
\text { thigh } \\
\text { pain and } \\
\text { swelling not } \\
\text { associated } \\
\text { with } \\
\text { fever or } \\
\text { abdominal } \\
\text { pain for } 2 \\
\text { weeks }\end{array}$ & $\begin{array}{l}\text { Normal } \\
\text { muscle } \\
\text { enzymes } \\
\text { ESR=I5 CRP: } \\
47 \text { ASOT: } \\
\text { normal } \\
\text { Virology \& } \\
\text { ANA negative }\end{array}$ & $\begin{array}{l}\text { subcutaneous } \\
\text { tissue dema } \\
\text { with intact } \\
\text { musculature }\end{array}$ & $\begin{array}{l}\text { patchy } \\
\text { myopathy }\end{array}$ & & $\begin{array}{l}\text { Heterozygous } \\
\text { for (M694V, } \\
\text { EI48Q , P369S) }\end{array}$ & typical & $+v e$ & $\begin{array}{l}\text { Steroids } \\
\mathrm{Img} / \mathrm{kg} / \mathrm{d} \text { for } \\
10 \text { weeks } \\
\text { began to } \\
\text { improve after } \\
\text { I month of } \\
\text { treatment }\end{array}$ \\
\hline
\end{tabular}

I. Obligatory criteria:

a. FMF-Prior clinical and/or genetic evidence of FMF or familial history of FMF.

b. Myalgia-Bilateral, of the lower and/or upper limbs, characterized by severe muscle pain and tenderness with physical disability, in the presence of normal muscle enzyme levels.

c. Duration of symptoms-Persistence of the myalgia for $\geq 5$ days.

II. Supporting criteria:

a. Homozygocity/hemizygocity for M694V mutation.

b. Elevated levels of inflammation markers: $\mathrm{ESR} \geq 80 \mathrm{~mm} / \mathrm{h}$, $\mathrm{CRP} \geq 5 \mathrm{mg} \%$.

c. Fever $\geq 38.0^{\circ} \mathrm{C}$.

III. Exclusion criteria:

a. Polyarteritis nodosa(PAN)

PFMS is thought to be a vasculitic process although the supporting pathologic evidence is lacking, ${ }^{19}$ Interestingly, other vasculitides such as Henoch Schonlein, Bechet's disease, and poly-arteritis nodosa have been found in an increased incidence among FMF patients. ${ }^{20}$ The exact mechanism describing the pathophysiology of PFMS is unknown, but there may be triggering factors such as an infection, or an interaction with other proteins induced by stress, trauma or hyperthermal balneotherapy, ${ }^{21}$ these factors regulate the immune response evolving in to an inflammatory state of PFMS in some FMF patients. ${ }^{22}$

Although colchicine is the cornerstone of FMF treatment, it has clearly been non effectious for PFMS. Corticosteroids is the mainstay in treatment. Though with high-dose steroids elicits resolution of symptoms in 5-7days, ${ }^{18}$ It may need prolonged time of intake more than 6 weeks for good response. ${ }^{23}$ Moreover, corticosteroids may not be beneficial in some patients, thus the use of other immunosuppressive was issued in various cases. ${ }^{24}$ To date, immunomodulatory agents like interferon, and tumor necrosis factor inhibitors have been employed in refractory \& severe cases of FMF with variable success but not issued in PFM perse. ${ }^{25}$

Demonstration of pivotal role of interleukin-1 (IL-1) in the pathogenesis of FMF leads to the use of IL-1 blockers in colchicine resistant or intolerant patients, Interleukin-1 (IL-1) antagonists, as anakinra, canakinumab and rilon accept are reported to be effective in colchicine unresponsive FMF patients. ${ }^{26-27}$

After reviewing the literatures; all reported cases of adolesecence and adult PFM and our case are summarized in Table 2. ${ }^{[13,18,21-23,28-33]}$ Up to that time we found 13 cases of FMF developed PFM. Their age range $12-44$ yrs with mean age $28 \pm 16$. Five cases of them were males $(38.46 \%) \& 8(61.53 \%)$ females. Five of them with documented positive family history $(38.46 \%)$ and one patient $(7.69 \%)$ with 
reported negative family history. Nine $(69.23 \%)$ patients were known to have FMF before the emerging the PFM while the rest of patients $(30.7 \%)$ diagnosed to have FMF at the onset of presentation. All patients shared the typical muscle ach and tenderness, only 8 of them; fever was associated. Normal muscle enzymes with high ESR \& CRP were the remarkable lab in diagnosis of all PFM cases. Three of the cases $(23.07 \%)$ were positive hetregonous for M694V and V726A; three $(23.07 \%)$ were positive homozygos for M694V while one patient $(7.69 \%)$ harboured homogenous for E148Q and compound heterogenous P3695-R408Q; while two case(14.38\%) beard positive heterozygous mutation E148 and R764.

Out of the cases, three (23.07\%) only underwent musckuloskeletal MRI on the affected muscles which revealed subcutanoeus and muscle oedema, EMG was done in four cases(30.7\%) [normal EMG findings done in $3(23.07 \%)$ cases only, non specific changes in one patient $(7.69 \%)$. Muscle biopsy was taken in 2 patients which demonstrated histological examination of the striated muscle did not show signs of vasculitis. There was an increase of the collagen tissue in the interstitium of the muscle and also a few inflammatory cells. The electron microscopic examination showed most muscle fibres were of normal size, but atrophic muscle cells of 17-20 im were dispersed. The sarcomere pattern was well preserved. The perivascular basement membrane of capillaries and of small venules was uniformly and moderately widened and the interstitium was infilterated with fibroblasts, macrophages, and a few mast cells. ${ }^{16}$

Mostly of the patients $[10(76.9 \%)]$ responded well to steroids while 2 cases showed steroid resistence and achieved proper improvement on the use of an IL-1 inhibitor, anakinra. Owing to the scarcity of studies about the management of steroid resistant PFM, we hereby declare the urgent need to evaluate the management of PFM \& variable response to different medication which might be related to phenotypic characteristics such as age of onset, ethnicity, Clinical presentation as well as frequency \& the severity of the attacks aside of the genotypic features. Herein, we reported a successful treatment with prolonged use of high dose steroids along 10 weeks with extensive physiotherapy

\section{Conclusion}

FMF can be considered to be recognized easily with the classical features including abdominal pain, fever, pleuritis, arthralgia \& myalgia. The atypical presentation of PFMS with severe disabling muscle pain and prolonged weakness may be a rare pattern of FMF. We would like to point out that FMF with PFM which respond well to steroids \& immunosuppressive drugs to reduce the time of pain and complications

\section{Acknowledgments}

None.

\section{Conflict of interests}

The author declares there is no conflict of interest.

\section{References}

1. Eliakim M, Levy M, Ehrenfeld M. Recurrent polyserositis. AmsterdamNew York-Oxford: Elsevier/North-Holland Biomedical Press; 1981:15.

2. Majeed HA, Al-Qudah AK, Qubain H, et al. The clinical patterns of myalgia in children with familial Mediterranean fever. Semin Arthritis Rheum. 2000;30(2):138-143.

3. Langevitz P, Zemer D, Livneh A, et al. Protracted febrile myalgia in patients with familial Mediterranean fever. $J$ Rheumatol. 1994;21(9):1708-9.

4. Livneh A, Langevitz P. Diagnostic and treatment concerns in familial Mediterranean fever. Baillieres Best Pract Res Clin Rheumatol. 2000;14(3):477-498.

5. Bakkaloglu A. Familial Mediterranean fever. Pediatr Nephrol. 2003;18(9):853-859.

6. The International FMF Consortium. Ancient missense mutations in a new member of the RoRet gene family are likely to cause familial Mediterranean fever. Cell. 1997;90(4):797-807.

7. Ben-Cherit E. Familial Mediterranean fever (FMF) and renal AA amyloidosis phenotype-genotype correlation, treatment and prognosis. J Nephrol. 2003;16(3):431-4.

8. Ben-Cherit E, Levy M. Familial Mediterranean fever. Lancet.1998;351:659-664.

9. Touitou I. The spectrum of familial Mediterranean fever (FMF) mutations. Eur J Hum Genet. 2001;9(7):473-83.

10. Soylu A, Kasap B, Türkmen M, et al. Febrile myalgia syndrome in familial Mediterranean fever. J Clin Rheumatol. 2006;12(2):93-96.

11. Tekin M, Yalc,nnkaya F, Tümer N, et al. Vasculitis in children with familial Mediterranean fever. In: Sohar E, Gahni J, Pras M, eds. Proceedings of the 1st International Conference on FMF; Jerusalem; Tel Aviv: Freund; 1997:162-167.

12. Duru NS, Civilibal M, Karakoyun M, et al. Protracted febrile myalgia in two children with familial Mediterranean fever. Pediatr Int. 2010;52(3):137-40.

13. Soylu A, Kasap B, Türkmen M, et al. Protracted febrile myalgia syndrome in a patient with familial Mediterranean fever homozygous for the E148Q mutation. Semin Arthritis Rheum. 2008;38(2):161-2.

14. Livneh A, Langevitz $\mathrm{P}$, Zemer D, et al. The changing face of familial Mediterranean fever. Semin Arthritis Rheum. 1996;26(3):612-27.

15. Schwabe AD, Peters RS. Familial Mediterranean Fever in Armenians. Analysis of 100 cases. Medicine (Baltimore). 1974; 53(6):453-62.

16. Schapira D, Ludatscher R, Nahir M, et al. Severe myalgia in familial Mediterranean fever: clinical and ultrastructural aspects. Ann Rheum Dis. 1988;47(1):80-83.

17. Kaplan E, Mukamel M, Barash J, et al. Protracted febrile myalgia in children and young adults with familial Mediterranean fever: analysis of 15 patients and suggested criteria for working diagnosis. Clin Exp Rheumatol. 2007;25:S114-7.

18. Tekin M, Yalçinkaya F, Tümer N, et al. Clinical, laboratory and molecular characteristics of children with Familial Mediterranean Fever-associated vasculitis. Acta Paediatr. 2000;89(2):177-82.

19. Ozçakar ZB, Yalçınkaya F. Vascular comorbidities in familial Mediterranean fever. Rheumatol Int. 2011;31:1275-1281.

20. Ozdogan H, Arisoy N, Kasapcapur O, et al. Vasculitis in familial Mediterranean fever. $J$ Rheumatol 1997;24(2):323-7.

21. Alpayci M, Kandemir A, Korkmaz M. Protracted Febrile Myalgia Syndrome: A Case Report Focusing on Possible Environmental Triggers. Arch of Rheum. 2013;28(3):209-211.

22. Sidi G, Shinar Y, Livneh y, et al. Protracted febrile myalgia of familial Mediterranean fever. Mutation analysis and clinical correlations. Scand $J$ Rheumatol. 2000;29(3):174-6.

23. Tufan G, Demir S. Uncommon clinical pattern of FMF: protracted febrile myalgia Syndrome. Rheumatol Int. 2010;30(8):1089-90. 
24. Bircan Z. S teroid-resistant protracted febrile myalgia. Semin Arthritis Rheum. 2010;40(3):e1-2

25. Ozturk MA, Kanbay M, Kasapoglu B, et al. Therapeutic approach to familial Mediterranean fever: a review update . Clin Exp Rheumatol. 2011;29(4 Suppl 67):S77-86 .

26. Meinzer U, Q uartier P, A lexandra JF, et al. Interleukin-1 targeting drugs in familial Mediterranean fever: a case series and a review of the literature. Semin Arthritis Rheum. 2011;41(2):265-71.

27. Gul A, Ozdogan H, Erer B, et al. Efficacy and safety of canakinumab in adults with colchicine resistant Familial Mediterranean Fever. Arthritis Rheum. 2012;64(Suppl):322.

28. Bircan Z, Usluer H. Protracted Febrile Myalgia Mimicking Polyarteritis Nodosa. J Clin Rheumatol. 2008;14(4):222-225.

29. Kotevoglu1 F, Sahin1S, Oguz Ozkiris M, et al. Protracted febrile myalgia of familial Mediterranean fever. Clin Exp Rheumatol. 2004;22(Suppl. 34):S69-S70.

30. Fujikawa K, Migita K, Tsukada T, et al. Protracted Febrile Myalgia Syndrome in a Japanese Patient with Fasciitis Detected on MRI. Intern Med 53(24):2817-9.

31. Ertekin V, Ays M, Alp H, et al. Familial Mediterranean fever protracted febrile myalgia in children: report of two cases Rheumatol Int (2005)25:398-400

32. Ridvan M, Aynur T, Berivan B, et al. Rapid resolution of protracted febrile myalgia syndrome with anakinra:Report of two cases. Mod Rheumatol. 2014;458-459.

33. Senel K, Melikoglu MA, Baykal T, et al. Protracted febrile myalgia syndrome in familial Mediterranean fever. Mod Rheumatol. 2010;20(4):410-2. 